\title{
Carcinoma of the ampulla of Vater in a haemophiliac patient
}

\author{
J. CATFORD \\ M.A., M.B., B.Chir. \\ R. ZEEGEN \\ M.B., M.R.C.P.

\section{Summary} \\ A 42-year-old haemophiliac who presented with a \\ cholestatic jaundice and repeated gastro-intestinal \\ haemorrhage is described. The bleeding was initially \\ thought to be due to the severity of the haemophilia \\ itself rather than from any local cause and the jaundice \\ thought to be due to a serum hepatitis. The correct \\ diagnosis of ampullary carcinoma was eventually made \\ after numerous barium studies, fibre-optic endoscopy \\ and two laparotomies. A carcinoma was discovered \\ at a second endoscopy some 12 months after initial \\ presentation. Attention is brought to the rare assoc- \\ iation of ampullary carcinoma and haemophilia, and \\ to the difficulties involved in diagnosis.
}

\author{
I. M. Prosser \\ M.B., B.S., D.M.R.D., F.F.R. \\ E. NAIK \\ M.B.B.S., M.R.C.Path.
}

Departments of Gastroenterology, Radiology, and Haematology, Westminster Hospital, London, S.W.1

\section{Case report}

A 42-year-old male with severe haemophilia
(AHG levels 0-2\%) presented in July 1973 with jaundice preceded by 2 weeks of anorexia, rigors and abdominal discomfort. For several months before $N$ this he had been given multiple infusions of cryo-: precipitate for haemarthroses, soft tissue bleeding, $\vec{c}$ etc. Liver function tests showed a bilirubin of $8.2 \mathrm{~N}$ $\mathrm{mg} / 100 \mathrm{ml}$, alkaline phosphates $216 \mathrm{KA}$ units and SGOT 120 iu (Fig. 1). In spite of a negative Australia antigen and the marked cholestasis, the most likely ${ }_{\mathbb{D}}$ diagnosis was considered to be a serum hepatitis. 을 Over the next 2 weeks the haemoglobin fell to $4.8 \mathrm{~g} / 100 \mathrm{ml}$ and this was thought to be due to occult $\mathrm{t}^{2}$ gastro-intestinal bleeding.

Since the age of 12 there had been thirteen episodês के of spontaneous gastro-intestinal bleeding, sorme requiring blood transfusion and on this occasion there was no history of dyspepsia, aspirin ingestion

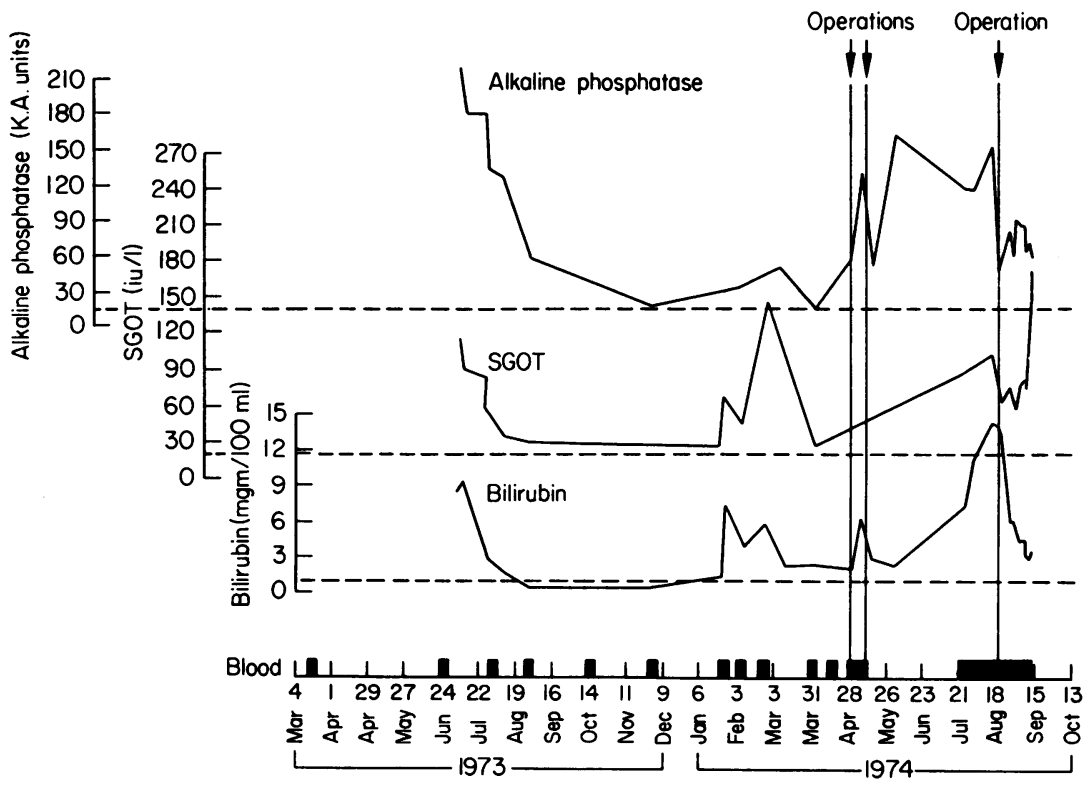

Fig. 1. Liver function tests from presentation. - - -, Upper limit of normal. 
or excessive alcohol. On admission he was anaemic, jaundiced, and the liver was smoothly enlarged to 2 fingers-breadths and slightly tender. He had multiple scars from cut-downs and transfusion sites and partial ankylosis of elbows and knees from repeated haemarthroses. Barium meal and followthrough, and enema were normal. A coned view of the gall-bladder area revealed no gall-stones and he was treated conservatively with blood and cryoprecipitate. Over the subsequent 5 months there were a further four similar episodes, each requiring blood and cryoprecipitate. During an admission in December 1973, melaena stools were noted for the first time. The liver function tests gradually improved, as shown in Fig. 1, but an auto-antibody screen revealed a strongly positive smooth muscle antibody and negative anti-mitochondrial antibody. A repeat barium meal was negative, but the deranged liver function tests did not allow radiological visualization of the biliary tree.

In January 1974, after a further haemorrhage, endoscopy of the upper gastro-intestinal tract was carried out with the 'Olympus' G.I.F.D. instrument, under cryoprecipitate cover. The oesophagus was normal, there were a number of small gastric erosions but the pylorus was too narrow to admit the instrument through into the duodenum. The duodenal cap, however, seen through the pylorus, appeared normal.

By April, after a transient rise in bilirubin and enzymes (Fig. 1), the liver functions again settled and there were no symptoms referable to the liver or biliary systems. However, melaena stools continued and even during those periods in which there was no overt bleeding, occult blood testing was positive. Quantification of the loss by labelling red cells with ${ }^{51} \mathrm{Cr}$ and counting the stools showed the blood loss was intermittent, $17 \mathrm{ml}$ being lost on one day but nil on a further three days. After a further three haemorrhages requiring transfusion, the patient was transferred to a haemophilia centre for diagnostic laparotomy and liver biopsy. The laparotomy under AHG cover on 3 May showed a superficial gastritis and a truncal vagotomy and antrectomy were carried out. Histology of the resected specimen showed a gastritis and the liver biopsy was reported as compatible with chronic persistent hepatitis or sclerosing cholangitis. Twelve days later, following repeated haematemeses, the abdomen was re-opened and the anastomosis line, which was bleeding, was resutured. His subsequent course was satisfactory, although the liver functions were again deranged, particularly the alkaline phosphatase, which at discharge was $163 \mathrm{KA}$ units.

Six weeks later there was a recurrence of gastrointestinal haemorrhage and a marked exacerbation of jaundice, as shown in Fig. 1. During the bleeding a further endoscopy was carried out. The instrument could now easily be introduced into the duodenum and a large, bleeding polypoid tumour was seen partially obstructing the lumen of the second part of the duodenum. This was thought to be an ampullary carcinoma and, at subsequent hypotonic duodenography (Fig. 2), the presence of an irregular peri-ampullary mass was confirmed. In fact, on careful review of two previous barium meals reported as negative, the lesion could be seen in the second part of the duodenum.

Diathermy excision of the ampullary carcinoma was carried out under AHG cover and there was no evidence of metastatic spread. Histology of the specimen (Fig. 3), which measured approximately $3 \mathrm{~cm} \times 3 \mathrm{~cm}$, was of a well-differentiated adenocarcinoma. Secondary haemorrhage began on the tenth post-operative day and persisted for almost 3 weeks, in spite of intensive cryoprecipitate and AHG therapy. Thereafter, however, the postoperative course was uncomplicated and he was discharged fit. Recent follow-up has been satisfactory although liver function tests remain abnormal (Fig. 1).

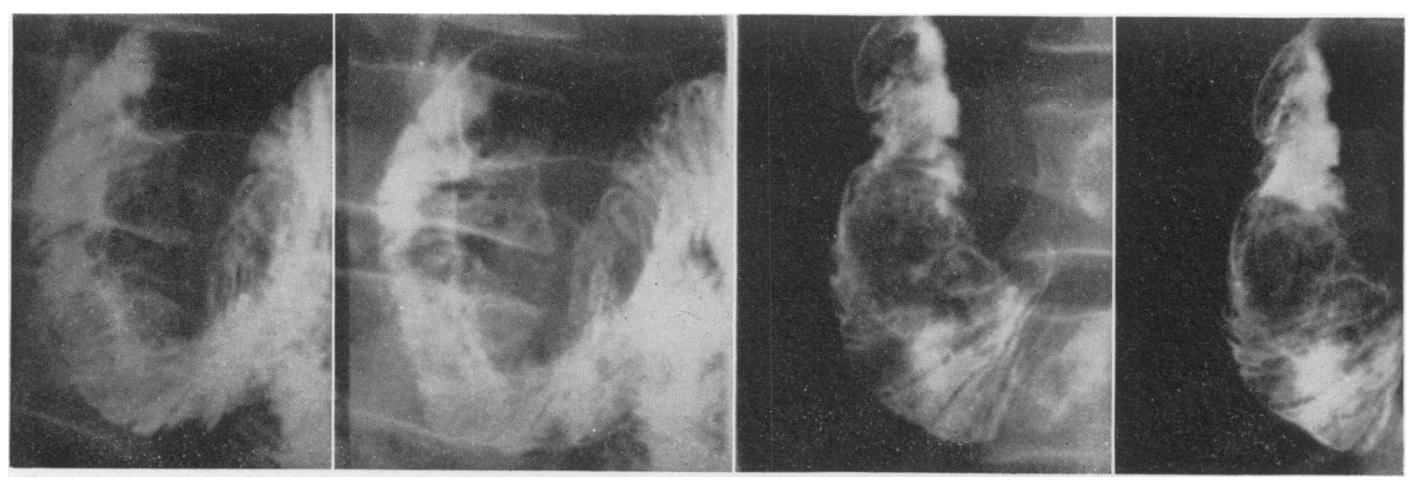

FIG. 2. Hypotonic duodenogram showing tumour. 


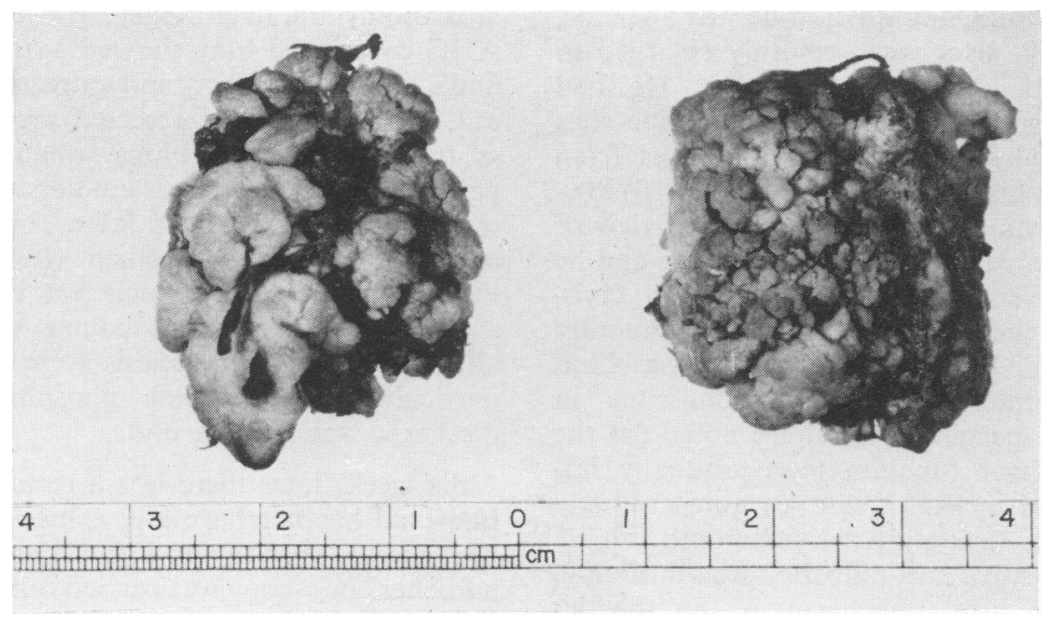

FIG. 3. Excised ampullary carcinoma.

\section{Discussion}

Although both gastro-intestinal haemorrhage and jaundice are well recognized in haemophilia (Wilkinson et al., 1961; Stuart et al., 1966; Forbes et al., 1973; Biggs, 1974), so far as the authors are aware this combination due to the association of carcinoma of the ampulla of Vater and haemophilia has not been reported. Gastro-intestinal haemorrhage frequently occurs in severe haemophiliacs, particularly adults, with no local cause found (Forbes et al., 1973) and in those mild haemophiliacs with bleeding, a dyspeptic history and a peptic ulcer is usually found. Jaundice in haemophilia is likely to be due to a serum hepatitis, and Biggs (1974) reported an average post-transfusion hepatitis incidence of $1.83 \%$ annually in a large national survey of treated haemophiliacs. The incidence of jaundice in 1837 patients with bleeding disorders (1625 with haemophilia) over a 3-year period was $3.48 \%$.

In this patient from presentation to diagnosis virtually 12 months had elapsed. Some 800 units of cryoprecipitate, 144 bottles of AHG and some 94 units of blood had been given. In addition to the severe haemophilia per se, the history suggestive of hepatitis without any clinical features of extrahepatic obstruction, negative barium studies, gastric erosions shown at endoscopy, strongly positive smooth muscle antibodies and a more or less complete resolution of abnormal liver functions, were factors favouring a continued conservative approach and contributing to the delay in diagnosis. Complete return to normality of disordered liver function is a recognized pitfall in the diagnosis of ampullary carcinona and, furthermore, this is a condition occurring in the elderly, $59 \%$ between the ages of $\mathbb{D}$ 50 and 69 (Sherlock, 1968). Percutaneous liver biopsy is clearly contra-indicated in haemophilia, but this was carried out as an open procedure at the first laparotomy. In any case liver biopsy intepretation can be extremely difficult and changes extra-hepatic obstruction can be misinterpreted, happened in this case.

Fibre-optic endoscopy was particularly valuable and is being used increasingly with good results to diagnose the site of gastro-intestinal haemorrhage $\mathbb{Q}_{\alpha}^{\mathbb{D}}$ (Cotton et al., 1973). After the lesion was discovered, $\overrightarrow{\vec{O}}$ a careful review of the recent barium films demon-응 strated abnormalities of the duodenal loop. This is a difficult area for confident radiological diagnosis and hypotonic duodenography may help to clarify. doubtful abnormalities in this region (Martel, 3 Scholtens and Lim, 1969).

The dangers of using invasive techniques in severe haemophilia, a rare cause of jaundice, to- $\delta$ gether with an atypical sequence of liver function $₹$ tests, delayed the diagnosis of ampullary carcinomao in this patient. The possibility of such a lesion should be borne in mind, even in relatively young haemo-o philiacs.

\section{Acknowledgment}

We thank Professor Harold Ellis for his advice and encouragement.

\section{References}

Biggs, R. (1974) Jaundice and antibodies directed against $\stackrel{\mathbb{Q}}{\mathscr{C}}$ factors VII and IX in patients treated for haemophilia or Christmas disease in the United Kingdom. British Journal of Haematology, 26, 313. 
Cotton, P.B., Rosenberg, M.T., Waldram, R.P.L. \& Axon, A.T.R. (1973) Early endoscopy of oesophagus, stomach and duodenal bulb in patients with haematemesis and melaena. British Medical Journal, 2, 505.

Forbes, C.D., Barr, R.D., Prentice, C.R.M. \& Douglas, A.S. (1973) Gastrointestinal bleeding in haemophilia. Quarterly Journal of Medicine, N.S., XLII, 503.

Martel, W., Scholtens, P.A. \& Lim, L.W. (1969) Tubeless hypotonic duodenography: Technique value and limitations. American Journal of Roentgenology, 107, 119.
SHERLOCK, S. (1968) Diseases of the Liver and Biliary System, 4th edition, 758-766. Blackwell Scientific Publications, Oxford.

Stuart, J., Davies, S.H., Cumming, R.A., Girdwood, R.H. \& DARG, A. (1966) Haemorrhagic episodes in haemophilia-a 5 year prospective study. British Medical Journal, 2, 1624.

Wilkinson, J.F., Nour-Eldin, F., IsRaels, M.C.G. \& BARRETT, K.E. (1961) Haemophilia syndromes. A survey of 267 patients. Lancet, ii, 947.

\title{
Pancreatitis and hyperparathyroidism
}

\author{
R. D. Rosin \\ M.B., B.S., F.R.C.S.(Ed), F.R.C.S. \\ Professorial Surgical Unit, Westminster Hospital, London
}

\begin{abstract}
Summary
Three patients with acute pancreatitis were found also to have hyperparathyroidism, two of them also formed pancreatic cysts. There appears to be more than an incidental relationship between the two diseases. The importance of serum calcium estimations in patients with pancreatitis is stressed. Theories as to why these diseases co-exist are reviewed.
\end{abstract}

\section{Introduction}

Hyperparathyroidism is one of the hormonal conditions that may be an aetiological factor in pancreatitis. However, the precise frequency with which pancreatitis occurs in association with hyperparathyroidism is not known. Mixter, Keynes and Cope (1962) found sixty-two cases of these diseases co-existing and reported that pancreatitis occurred in eleven out of 155 patients with hyperparathyroidism. It appears that either the coexistence of these two diseases is not as common in the United Kingdom as in the United States of America, or that pancreatitis is not investigated so thoroughly to exclude hyperparathyroidism.

\section{Case 1}

A 72-year-old man was admitted in December 1971 to Sutton Hospital as an emergency with a sudden onset of colicky abdominal pain which did not radiate to his back. He gave no history of indigestion, vomiting, change of bowel habit or change in colour of his stools or urine. He was known to have chronic bronchitis and emphysema and cardiographic evidence of ischaemic heart disease. He smoked twenty cigarettes per day and admitted to a moderate alcohol intake.

On examination he was an ill looking man with a pulse rate of $92 / \mathrm{min}$. His blood pressure was $200 / 110$ $\mathrm{mmHg}$. He was apyrexial, and his tongue was dry and coated. The thyroid gland was not enlarged. There was guarding and tenderness across the upper abdomen. The results of the main investigations performed were: haemoglobin $11.9 \%$; white blood count $15.000 \mathrm{~mm}^{3}$; serum amylase 3700 Somogyi units; urea and electrolytes were within normal limits. Radiological examination showed a calcified pleural plaque on the left side with a small pneumothorax. An X-ray of the abdomen was normal, and there were no pancreatic calculi.

A diagnosis of pancreatitis was made, and the patient was treated with intravenous fluids, papaveretum $20 \mathrm{mg}$, ampicillin $500 \mathrm{mg}$, and trasylol 25,000 u 6 hourly, the latter administered via the infusion and continued for $\mathbf{8}$ days. On the following day his condition was unchanged, although he felt more comfortable. Bruising was present in his left flank (Grey Turner's sign). The serum calcium was measured and found to be $13.4 \mathrm{mg} / 100 \mathrm{ml}$ (normal range $8.5-10.5 \mathrm{mg} / 100 \mathrm{ml}$ ). Forty-eight hours after admission he became shocked and had an irregular tachycardia of $116 / \mathrm{min}$ with profuse sweating. He was digitalized and the diagnosis of acute pancreatitis was confirmed by a peritoneal aspiration which showed typical blood-stained plum-coloured fluid. 